\title{
Dehydroepiandrosterone Sulfate Level Varies Nonlinearly with Symptom Severity in Major Depressive Disorder
}

\author{
Dasom Uh ${ }^{1}$, Hyun-Ghang Jeong ${ }^{1,2}$, Kwang-Yeon $\mathrm{Choi}^{1}$, So-Young $\mathrm{Oh}^{3}$, Suji Lee ${ }^{4}$, Seung-Hyun Kim ${ }^{1}$, \\ Sook-Haeng Joe J,2 $^{1,-1}$ \\ ${ }^{1}$ Department of Psychiatry, Korea University Guro Hospital, Korea University College of Medicine, ${ }^{2}$ Research Institute of Mental Health, Korea \\ University, ${ }^{3}$ Department of Psychiatry, Seoul Metropolitan Eunpyeong Hospital, ${ }^{4}$ Department of Biomedical Sciences, Korea University Graduate \\ School, Seoul, Korea
}

\begin{abstract}
Objective: The pathophysiology of major depressive disorder (MDD) is still not well understood. Conflicting results for surrogate biomarkers in MDD have been reported, which might be a consequence of the heterogeneity of MDD patients. Therefore, we aim to investigate how the severity of depression and various symptom domains are related to the levels of dehydroepiandrosterone sulfate (DHEA-s) in MDD patients.

Methods: We recruited 117 subjects from a general practice. Depressive symptoms were assessed using the Beck Depression Inventory (BDI). Depressive symptoms were divided into three subdomains according to BDI items; somatic symptoms, guilt and failure, and mood and inhibition.

Results: In subjects with very-mild-to-moderate depression, the DHEA-s level increased as BDI score did. However, the DHEA-s levels in the subjects with severe depression were significantly lower than in subjects with moderate depression $(p=0.003)$. DHEA-s level was correlated with the BDI subscore for guilt and failure in very-mild-to-moderate depression $(r=0.365, p=0.006)$.

Conclusion: The DHEA-s level appears to be indicative of MDD severity with respect to depressive symptoms, especially regarding guilt and failure. Our findings suggest that the upregulation of DHEA-s may be a part of a compensatory process in very-mild-to-moderate depression, and the failure of this compensation mechanism may underlie the development of severe depression.
\end{abstract}

KEY WORDS: Dehydroepiandrosterone sulfate; Depression; Differential diagnosis; Neuroendocrinology.

\section{INTRODUCTION}

Major depressive disorder (MDD) is a common mental health illness, with a lifetime prevalence estimated at $16.2 \%$ in the United States. ${ }^{1)}$ Development of MDD might be multifactorial; various psychosocial stressors, comorbid illnesses, and some drugs, especially in susceptible individuals having genetic predisposition, could lead to alterations in central monoaminergic transmission, abnormalities in neurotransmitter receptor function, dysregulation of hypothalamic-pituitary-adrenal (HPA) axis, decreased neurotrophic factors, increased pro-inflam-

Received: July 4, 2016 / Revised: September 14, 2016

Accepted: December 3, 2016

Address for correspondence: Hyun-Ghang Jeong, MD, PhD Department of Psychiatry, Korea University Guro Hospital, Korea University College of Medicine, 148 Gurodong-ro, Guro-gu, Seoul 08308, Korea

Tel: +82-2-2626-3164, Fax: +82-2-852-1937

E-mail: jeonghg@korea.ac.kr matory cytokines, increased oxidative stress, etc. ${ }^{2-8)}$ Many potential surrogate markers in peripheral blood reflecting such neurobiological changes have been explored and tested for their value in predicting the diagnosis and prognosis of MDD. ${ }^{9,10)}$

Dehydroepiandrosterone sulfate (DHEA-s) has been draw attention as an one of the potential surrogate markers related to pathophysiology of the MDD. ${ }^{11)}$ DHEA-s is neuroactive steroid and distributes in both central nervous system and peripheral circulation. Higher DHEA-s level is likely to have some beneficial effects on improving attention ${ }^{12)}$ and lowering perceived stress. ${ }^{13,14)}$ Some studies suggests that higher DHEA-s and DHEA may counteract the neurotoxic effects due to high cortisolemia in depression. ${ }^{15,16)}$

Some conflicting results concerning associations between MDD and mediating factors including DHEA-s bring us to reconsider methodological issues in research. Diagnostic criteria of MDD may incorporate heteroge-

(C) This is an Open-Access article distributed under the terms of the Creative Commons Attribution Non-Commercial License (http://creativecommons.org/licenses/by-nc/4.0) which permits unrestricted non-commercial use, distribution, and reproduction in any medium, provided the original work is properly cited. 
neous patients into one group. ${ }^{17)}$ MDD patients could show different severity of symptoms. Each category of mild, moderate, and severe MDD patients may involve different neurobiological changes. Some neurobiological changes observed in MDD patient might be state dependent. ${ }^{18,19)}$ Thus, MDD patients present a broad spectrum of symptoms; somatic symptoms, cognitive symptoms, and neurovegetative symptoms, along with depressive mood or loss of interest. Different symptoms of MDD are likely to have different links to various neurobiological changes. ${ }^{20,21)}$ Appropriately specifying MDD into subtypes thus helps us to expand our understanding of the neurobiology of MDD.

Therefore, this study aims to investigate the relationships between different degrees of severity and different symptom domains of MDD and DHEA-s. We hypothesized that DHEA-s would be lower in patients with severe depression than in those with mild depression. Peripheral DEHA-s level would also be different in relation to the subodomain of major depressive symptoms (somatic, guilt and failure, or mood and inhibition). Our secondary objective is to investigate other putative markers for dpression such as cortisol, folate, homocysteine, and lipid profiles in peripheral blood. Cortisol seems to be related to the HPA axis dysfunction in depressive patients. ${ }^{22)}$ Plasma folate and homocysteine may be associated with oxidative stress in depression. ${ }^{23)}$ Dyslipidemia may mediate the development of depressive symptoms and increase the risk for depression. ${ }^{24)}$

\section{METHODS}

\section{Subjects}

Patients with MDD were recruited from the outpatient clinic of Korea University Guro Hospital from June 2013 to August 2014. The patients were diagnosed through semi-structured clinical interviews, which included the questions from the Mini-International Neuropsychiatric Interview (MINI) module based on the DSM-5, by one psychiatrist. Further probing regarding other comorbid psychiatric illnesses was prompted if necessary. All subjects were psychotropic drug-free for two weeks prior to study enrollment. We excluded patients with substance abuse or dependence, bipolar disorder, or other psychotic disorders. Subjects with neurological or relevant endocrinological disorders and those taking hormone replacement therapy were also excluded. Among the patients, 117 subjects who fulfilled the inclusion criteria underwent blood sampling and completed self-report questionnaires about their depressive symptoms. A questionnaire on demographic and health-related information was administrated to survey subjects' smoking, alcohol intake, current medications, height, weight, and other factors. All participants were fully informed of the study protocol, which was approved by the Institutional Review Board of the Korea University Guro Hospital (No. KUGH14118).

\section{Blood Samples}

Morning blood samples were collected from all patients between 08:00 am and 10:00 am. Blood samples were centrifuged immediately, and serum was stored at $-20^{\circ} \mathrm{C}$ until they were used in assay. These samples were assayed for levels of DHEA-s and cortisol using a ${ }^{125}$ I-radioimmunoassay method. DHEA-s plasma level was tested with the TKDS-2 DHEA SO4 Coat-A-Count ${ }^{\mathbb{R}}$ kit (Siemens Healthcare Diagnostics, Tarrytown, NY, USA) according to manufacturer directions. Cortisol was measured with the TKCO-11 Coat-A-Count kit (Siemens Healthcare Diagnostics). The inter- and intra-assay coefficients of variation for DHEA-s and cortisol were $11.0 \%$ and $5.1 \%$. DHEA-s was chosen over DHEA because of its absence of diurnal variation, much higher blood levels, and longer half-life. ${ }^{25)}$ Total homocysteine was measured by chemiluminescent microparticle immunoassay. ARCHITECT Homocysteine Reagent kits (Abbott GmbH \& Co. KG, Wiesbaden, Germany) were used on the ARCHITECT $i$ system. Folate level was determined by radioassay on MP Biomedicals SimulTRAC-SNB Radioassay kit (ICN Pharmaceuticals, Costa Mesa, CA, USA).

\section{Assessment of Depressive Symptoms}

Depressive symptoms were assessed using the Beck Depression Inventory (BDI). The BDI is one of the most widely used instruments to measure the severity of depression. It is a self-report questionnaire that contains 21 items asking about vegetative, emotional, cognitive, and motivational symptoms associated with depression. The Korean version of the BDI has been previously validated. ${ }^{26)}$

The scoring methods used in the Korean BDI are Likert-style $(0,1,2,3)$, with total score ranging from 0 to 63; a higher total score reflects more severe depressive symptoms. Subjects were classified by total score on the BDI: 9 or less as having very mild depression, 10 to 15 mild depression, 16 to 23 moderate depression, and 24 to 63 severe depression. Further, Bouman and $\mathrm{Kok}^{27)}$ proposed a division of the BDI into three subscales; 1 ) somatic, 2) guilt and failure, and 3) mood and inhibition. 
Table 1. Descriptive statistics for demographic and social variables

\begin{tabular}{|c|c|c|c|c|c|}
\hline Variable & Very mild & Mild & Moderate & Severe & $p$ value \\
\hline Patient & $7(6.0)$ & $22(18.8)$ & $27(23.1)$ & $61(52.1)$ & \\
\hline Age $(y r)$ & $55.86 \pm 9.35$ & $50.18 \pm 20.29$ & $52.37 \pm 14.37$ & $58.80 \pm 12.01$ & 0.069 \\
\hline Sex & & & & & 0.058 \\
\hline Male & 57.1 & 31.8 & 48.1 & 23.0 & \\
\hline Female & 42.9 & 68.2 & 51.9 & 77.0 & \\
\hline Education (yr) & $11.17 \pm 5.07$ & $10.36 \pm 4.15$ & $11.81 \pm 3.24$ & $8.20 \pm 4.58$ & 0.003 \\
\hline Body mass index $\left(\mathrm{kg} / \mathrm{m}^{2}\right)$ & $25.23 \pm 3.31$ & $23.48 \pm 2.33$ & $23.01 \pm 3.76$ & $23.40 \pm 3.56$ & 0.463 \\
\hline Alcohol $(\%)$ & & & & & 0.331 \\
\hline Never & 33.3 & 59.1 & 53.8 & 69.5 & \\
\hline Previously & 0 & 4.5 & 3.8 & 6.8 & \\
\hline Currently & 66.7 & 36.4 & 42.3 & 23.7 & \\
\hline Smoking (\%) & & & & & 0.278 \\
\hline Never & 83.3 & 77.3 & 65.4 & 78.0 & \\
\hline Previously & 16.7 & 13.6 & 11.5 & 3.4 & \\
\hline Currently & 0 & 9.1 & 23.1 & 18.6 & \\
\hline
\end{tabular}

Values are presented as number (\%), mean \pm standard deviation, or percentage only.

Each subscale score is calculated by summing the points on that subscale's items, as follows: The BDI somatic subscale (BDI-s) comprises BDI items 10, 11, 16, 17, 18, 19, 20, and 21; the BDI guilt and failure subscale (BDI-gf) comprises BDI items 3, 5, 7, and 8; and the BDI mood and inhibition subscale (BDI-mi) comprises BDI items 1, 2, 9, 13 , and 15. All subscales displayed adequate internal consistency reliability.

\section{Data Analysis}

We analyzed differences in demographic factors across the four depression-severity groups using the chi-squared test for categorical variables and the Kruskal-Wallis test for continuous variables. Furthermore, to assess the potential influence of each BDI subscale on the levels of the parameters, we separately assessed Pearson's correlations between each BDI subscale scores and those parameters that showed significant results. All statistical analyses were performed using the SPSS ver. 12.0 statistical package (SPSS Inc., Chicago, IL, USA).

\section{RESULTS}

\section{Sociodemographic Characteristics}

A total of 117 patients (79 female and 38 male) with MDD were enrolled in this study. Table 1 presents the patients' characteristics and demographic data. Subjects were aged 17 to 81 years (mean \pm standard deviation, $55.52 \pm 15.5)$. The mean BDI score was $25.3( \pm 11.6)$. Seven patients reported very mild depressive symptoms that were residual symptoms subsequent to past major depressive episodes. The subjects with mild, moderate, and

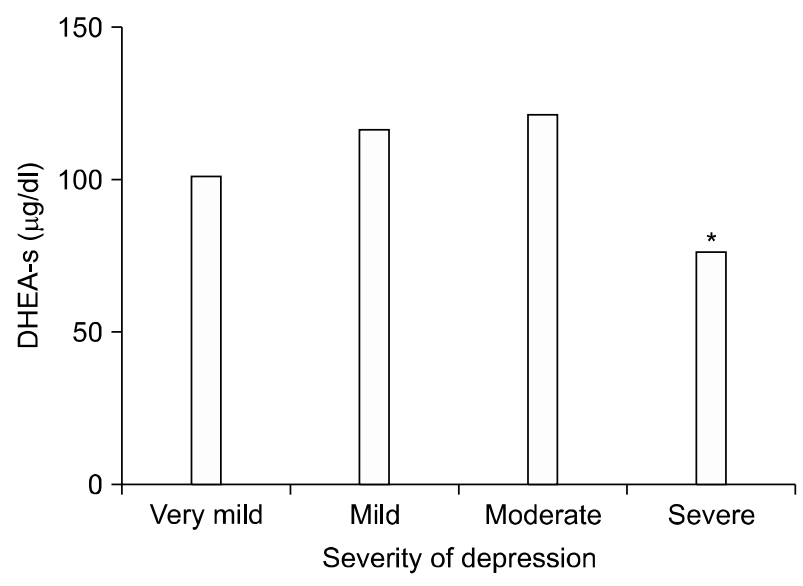

Fig. 1. Kruskal-Wallis test for dehydroepiandrosterone sulfate (DHEA-s) level in the groups of different severity of depression. The severe depression group had lower levels of DHEA-s than all other groups. *Moderate group vs. severe group $(p=0.003)$.

severe depression numbered 22, 27, and 61, respectively. There were no significant differences in age, sex, or BMI across severity groups.

\section{Relationship between Severity of Depressive Symptoms and DHEA-s Level}

The DHEA-s level did show significant differences among the depression-severity groups $(p=0.006)$. As shown in Figure 1, the mean DHEA-s level $(76 \pm 71 \mu \mathrm{g} / \mathrm{dl})$ of the subjects with severe depression was significantly lower than that $(120 \pm 79 \mu \mathrm{g} / \mathrm{dl})$ of subjects with moderate depression $(p=0.003)$. But among subjects with verymild-to-moderate depression, the DHEA-s level increased with the BDI score. However, this trend was not 
apparent in subjects with severe depression.

\section{DHEA-s Level and Depression Severity according to Different Subdomains of Depressive Symptoms}

We performed further analysis to identify whether the subdomain of depressive symptoms influenced the relationship between DHEA-s level and depression severity. We determined the correlation for the scores of each BDI subscale (somatic, guilt and failure, or mood and inhibition) with the DHEA-s level in two groups: the very-mild-to-moderate depression group and the severe depression group (Fig. 2). There was a significant positive correlation between DHEA-s level and BDI-gf in the very-mild-to-moderate group $(\mathrm{r}=0.365, p=0.006)$, but not in the severe group $(\mathrm{r}=0.057, p=0.663)$. No significant correlations with DHEA-s were found for the other two BDI subscores in either group: in the very-mild-to-moderate group $(\mathrm{r}=0.05, p=0.712$ for $\mathrm{BDI}-\mathrm{mi} ; \mathrm{r}=-0.228$, $p=0.09$ for BDI-s) or severe group ( $\mathrm{r}=0.113, p=0.387$ for BDI-mi; $\mathrm{r}=-0.187, p=0.148$ for BDI-s).
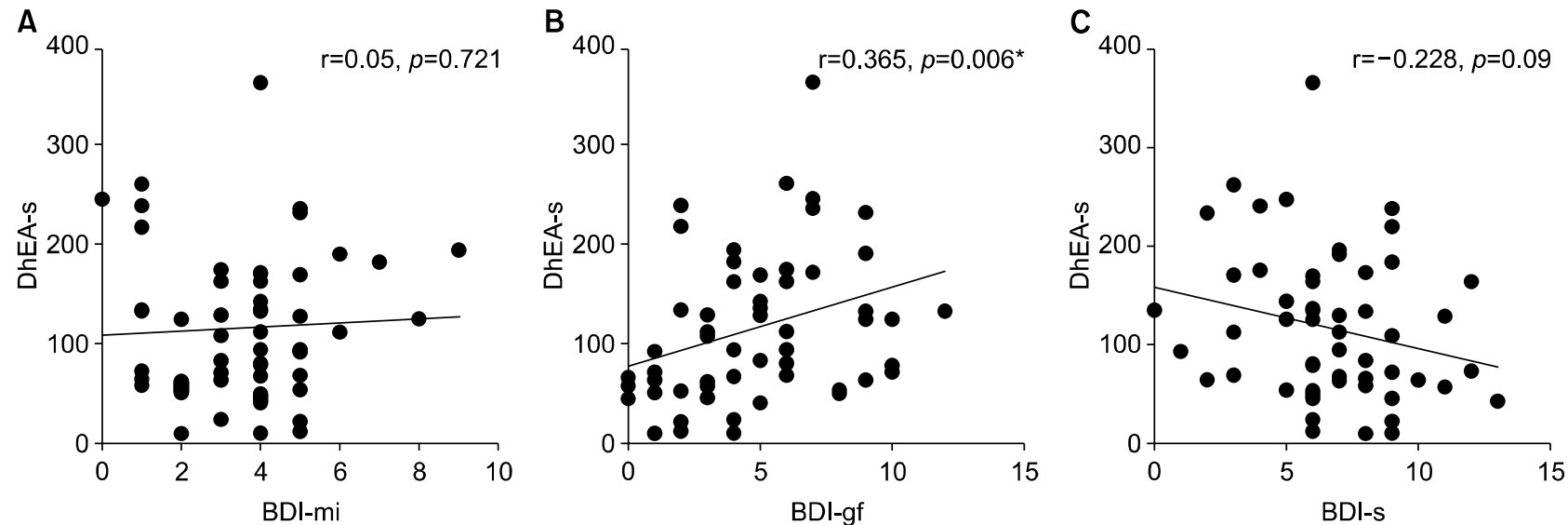

Very-mild-to-moderate group
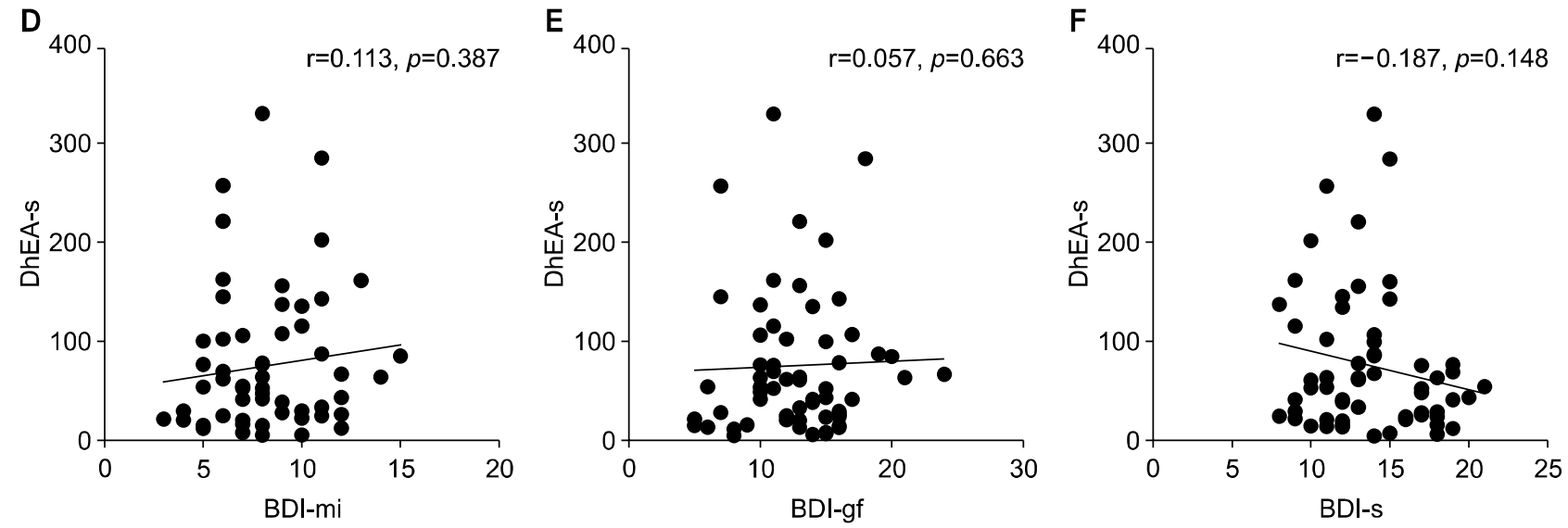

Fig. 2. Correlation between the dehydroepiandrosterone sulfate (DHEA-s) level and the Beck Depression Inventory (BDI) subscales. In the very-mild-to-moderate severity group ( $A, B$, and $C$ ), the dehydroepiandrosterone sulfate (DHEA-s) level is correlated with the score on the BDI-gf subscale $(r=0.365, p=0.006)$, but not in the severe group. No correlation was found between DHEA-s level and the other two BDI subscores.

BDI-mi, the BDI mood and inhibition subscale; BDI-gf, the BDI guilt and failure subscale; BDI-s, the BDI somatic subscale. 
Table 2. DHEA-s, cortisol, folate, homocysteine, and lipid profiles in patients with different degrees of severity of depressive symptoms

\begin{tabular}{|c|c|c|c|c|c|}
\hline & Very mild & Mild & Moderate & Severe & $p$ value \\
\hline DHEA-s $(\mu \mathrm{g} / \mathrm{dl})$ & $101.14 \pm 68.02$ & $116.18 \pm 73.16$ & $120.93 \pm 79.64$ & $76.13 \pm 71.52$ & 0.006 \\
\hline Cortisol ( $\mu \mathrm{g} / \mathrm{dl})$ & $11.83 \pm 3.19$ & $10.06 \pm 4.17$ & $10.30 \pm 5.38$ & $10.20 \pm 7.01$ & 0.285 \\
\hline Folate $(\mathrm{ng} / \mathrm{ml})$ & $11.80 \pm 3.96$ & $9.33 \pm 4.04$ & $10.32 \pm 6.03$ & $10.37 \pm 3.96$ & 0.556 \\
\hline Homocystein $(\mu \mathrm{mol} / \mathrm{L})$ & $9.71 \pm 2.43$ & $9.70 \pm 3.73$ & $9.05 \pm 2.52$ & $9.79 \pm 3.85$ & 0.780 \\
\hline $\mathrm{HDL}(\mathrm{mg} / \mathrm{dl})$ & $54.17 \pm 20.39$ & $57.17 \pm 12.70$ & $49.76 \pm 13.95$ & $53.46 \pm 12.11$ & 0.348 \\
\hline $\mathrm{LDL}(\mathrm{mg} / \mathrm{dl})$ & $122.67 \pm 33.33$ & $110.61 \pm 37.52$ & $102.86 \pm 30.08$ & $113.43 \pm 33.15$ & 0.435 \\
\hline Triglyceride (mg/dl) & $156.50 \pm 69.22$ & $116.61 \pm 54.60$ & $126.24 \pm 69.84$ & $163.94 \pm 135.02$ & 0.397 \\
\hline Cholesterol (mg/dl) & $191.60 \pm 49.26$ & $185.06 \pm 42.24$ & $169.30 \pm 38.27$ & $189.65 \pm 40.54$ & 0.279 \\
\hline Total lipid (mg/dl) & $655.83 \pm 117.56$ & $604.06 \pm 117.53$ & $579.40 \pm 110.17$ & $631.19 \pm 163.78$ & 0.349 \\
\hline
\end{tabular}

Values are presented as mean \pm standard deviation.

DHEA-s, dehydroepiandrosterone sulfate; HDL, high density lipoprotein; LDL, low density lipoprotein.

\section{Other Neuroendocrine Data and Lipid Profile}

Levels of plasma cortisol, homocysteine, and folate did not differ significantly across the four groups categorized by depression severity. There were also no significant differences in mean plasma concentrations of high density lipoprotein (HDL), low density lipoprotein (LDL), triglycerides, cholesterol, or total lipids across the severityof-depression groups (Table 2).

\section{DISCUSSION}

This study elucidates the association between serum DHEA-s level and the severity of depressive symptoms and psychometric properties in MDD patients. DHEA-s level differed significantly across the degrees of severity of depressive symptoms: DHEA-s increased with severity of depressive symptoms measured by the BDI in very-mild-to-moderate depression patients, but not in patients with severe depression, who had a significantly lower level. In further analysis, the relationship with DHEA-s level was apparent for MDD symptoms related to guilt and sense of failure, but not for somatic symptoms or those related to mood and inhibition.

Accumulated data suggests that DHEA-s is likely to have antidepressant effects. DHEA-s, a neuroactive steroid, has neurobiological actions through modulating the release of serotonin, dopamine, and norepinephrine. ${ }^{22,28-30)}$ Animal studies have revealed that DHEA-s administration improves depressive-like behavior, mediated by $\gamma$-aminobutyric acid receptors in the mesolimbic system. ${ }^{31)}$ In addition, anti-inflammatory and anti-oxidant properties of DHEA-s, along with various biological actions of DHEA-s in the brain, such as neurogenesis and inhibition of apoptosis, are possibly related to mechanisms against depression. ${ }^{22}$ Supporting these findings, some previous studies reported lower DHEA-s levels in patients with depression than in normal controls. ${ }^{32,33)}$ However, other studies have shown increased DHEA-s concentrations in depressed patients, ${ }^{34,35)}$ or even no significant differences between those groups. ${ }^{36)}$ These discrepancies across studies might be attributed to the fact that these studies were conducted in heterogeneous populations in terms of the severity of depressive symptoms. As our results indicate, both patients with very-mild-to-moderate depressive symptoms and those with severe depressive symptoms showed distinct transitions in DHEA-s level. We suggest that the relationship between DHEA-s level and severity of depression is not a linear pattern, and that this might explain the previous inconsistent results.

We cautiously speculate that upregulation of DHEA-s level in the very-mild-to-moderate groups may be involved in a compensatory process to maintain a homeostasis against depressive symptoms. Studies investigating the effects of acute psychosocial stress on DHEA and DHEA-s levels have shown significantly elevated DHEA and DHEA-s levels in response to stress and have shown increases of DHEA concentration during stressful situations, which may contribute to reduction of negative mood. ${ }^{37,38)}$ Failure of this compensatory process in sustaining homeostasis may result in severe depressive symptoms. Our findings may help to explain previous conflicting findings, which may be due to the differing definitions of symptom severity and categories of depression among studies. In further analysis, the relationship between severity and DHEA-s level was observed specifically for depressive symptoms related to guilt and failure, but not for somatic symptoms or those related to mood and inhibition. Some previous studies that divided depressive symptoms into psychological and neurovegetative symptoms found DHEA-s level to be more closely related to psychological symptoms than neurovegetative symptoms. ${ }^{39-41)}$ There is evidence to suggest that psychological symptoms 
may involve alterations in serotonin metabolism, whereas vegetative symptoms may be affected by dopamine activity in the basal ganglia. It has been proposed that individual subdomains of depressive symptoms might be mediated by different pathophysiologic mechanisms. ${ }^{21)}$ Consistent with this view, our results suggest that DHEA-s also links differently for the different subdomains of depressive symptoms.

In contrast, previous studies also have reported significant association between DHEA and somatic symptoms in non-depression patients. ${ }^{42)}$ Estrogenic and androgenic derivatives of DHEA may interact with androgen and estrogen receptors to modulate physiologic effects such as sexual function, libido, and fatigue. ${ }^{43)}$ However, the relationship between DHEA-s and somatic symptoms in MDD patients is still unclear. Additional studies are needed for further clarification.

In this study, we failed to find the differences among cortisol, folate, homocysteine, and lipid profiles in peripheral blood between subjects with different severity of depressive symptoms. These putative markers might not be enough to reflect the differences of severity in depressive symptoms among subjects with MDD, and not be as sensitive as detecting the distinction between MDD subjects and healthy controls. Further study might be needed in larger population.

This study contributes to increasing insight regarding the role of DHEA-s in MDD. Our results suggest that the DHEA-s level in MDD patients changes according to the severity and specific subdomain of depressive symptoms. These findings bring important clinical implications. Peripheral DHEA-s could serve as a state marker of MDD symptoms. Additionally, our findings support an alternative use of exogenous DHEA administration, especially in severely depressed patients.

Along with these important clinical implications, a few limitations of this study should be considered. First, we analyzed only DHEA-s levels, and not DHEA data, in MDD patients. However, because DHEA-s levels remain relatively consistent during the day, whereas DHEA has diurnal variation, findings based on DHEA-s may be more robust than those based on DHEA. Second, the subjects did not include individuals without MDD because this observational study utilized clinical samples. We were therefore not able to present a direct comparison of DHEA-s level between those with MDD and healthy controls. Third, the sample size is relatively small for a study using multiple measures. Further investigation with larger samples and including healthy controls is necessary. Fourth, semi-struc- tured interview used in this study was not validated.

In conclusion, our findings suggest that peripheral DHEA-s reflects the severity of symptoms in MDD patients as well as the spcific depressive symptoms of guilt and failure. Increasing peripheral DHEA-s level may be a part of a compensatory process in very mild-to-moderate depression, and the failure of this compensation mechanism may be related to the development of severe depression. DHEA-s is a possible useful marker of severity and psychometric properties of MDD.

\section{REFERENCES}

1. Andrade L, Caraveo-Anduaga JJ, Berglund P, Bijl RV, De Graaf R, Vollebergh W, et al. The epidemiology of major depressive episodes: results from the International Consortium of Psychiatric Epidemiology (ICPE) surveys. Int $J$ Methods Psychiatr Res 2003;12:3-21.

2. Hasler G. Pathophysiology of depression: do we have any solid evidence of interest to clinicians? World Psychiatry 2010;9:155-161.

3. Sullivan PF, Neale MC, Kendler KS. Genetic epidemiology of major depression: review and meta-analysis. Am J Psychiatry 2000;157:1552-1562.

4. Savitz J, Lucki I, Drevets WC. 5-HT(1A) receptor function in major depressive disorder. Prog Neurobiol 2009;88:17-31.

5. Kathol RG, Jaeckle RS, Lopez JF, Meller WH. Pathophysiology of HPA axis abnormalities in patients with major depression: an update. Am J Psychiatry 1989;146:311-317.

6. Martinowich K, Manji H, Lu B. New insights into BDNF function in depression and anxiety. Nat Neurosci 2007; 10:1089-1093.

7. Leonard B, Maes M. Mechanistic explanations how cellmediated immune activation, inflammation and oxidative and nitrosative stress pathways and their sequels and concomitants play a role in the pathophysiology of unipolar depression. Neurosci Biobehav Rev 2012;36:764-785.

8. Charney DS. Monoamine dysfunction and the pathophysiology and treatment of depression. J Clin Psychiatry 1998;59 Suppl 14:11-14.

9. Schmidt HD, Shelton RC, Duman RS. Functional biomarkers of depression: diagnosis, treatment, and pathophysiology. Neuropsychopharmacology 2011;36:2375-2394.

10. Schneider B, Prvulovic D. Novel biomarkers in major depression. Curr Opin Psychiatry 2013;26:47-53.

11. Peixoto C, Devicari Cheda JN, Nardi AE, Veras AB, Cardoso A. The effects of dehydroepiandrosterone (DHEA) in the treatment of depression and depressive symptoms in other psychiatric and medical illnesses: a systematic review. Curr Drug Targets 2014;15:901-914.

12. Wolf OT, Neumann O, Hellhammer DH, Geiben AC, Strasburger CJ, Dressendörfer RA, et al. Effects of a two-week physiological dehydroepiandrosterone substitution on cognitive performance and well-being in healthy elderly women and men. J Clin Endocrinol Metab 1997;82: 2363-2367.

13. Morgan CA 3rd, Rasmusson A, Pietrzak RH, Coric V, Southwick SM. Relationships among plasma dehydroepiandrosterone and dehydroepiandrosterone sulfate, cortisol, symptoms of dissociation, and objective performance in humans exposed to underwater navigation stress. Biol 
Psychiatry 2009;66:334-340.

14. Russo SJ, Murrough JW, Han MH, Charney DS, Nestler EJ. Neurobiology of resilience. Nat Neurosci 2012;15:1475-1484.

15. Goodyer IM, Herbert J, Altham PM. Adrenal steroid secretion and major depression in 8- to 16-year-olds, III. Influence of cortisol/DHEA ratio at presentation on subsequent rates of disappointing life events and persistent major depression. Psychol Med 1998;28:265-273.

16. Kaminska M, Harris J, Gijsbers K, Dubrovsky B. Dehydroepiandrosterone sulfate (DHEAS) counteracts decremental effects of corticosterone on dentate gyrus LTP. Implications for depression. Brain Res Bull 2000;52:229-234.

17. Chen L, Eaton WW, Gallo JJ, Nestadt G. Understanding the heterogeneity of depression through the triad of symptoms, course and risk factors: a longitudinal, population-based study. J Affect Disord 2000;59:1-11.

18. Sarandol A, Sarandol E, Eker SS, Erdinc S, Vatansever E, Kirli S. Major depressive disorder is accompanied with oxidative stress: short-term antidepressant treatment does not alter oxidative-antioxidative systems. Hum Psychopharmacol 2007;22:67-73.

19. Thomas AJ, Davis S, Morris C, Jackson E, Harrison R, O'Brien JT. Increase in interleukin-1beta in late-life depression. Am J Psychiatry 2005;162:175-177.

20. Duivis HE, Vogelzangs N, Kupper N, de Jonge P, Penninx BW. Differential association of somatic and cognitive symptoms of depression and anxiety with inflammation: findings from the Netherlands Study of Depression and Anxiety (NESDA). Psychoneuroendocrinology 2013;38:1573-1585.

21. Capuron L, Miller AH. Cytokines and psychopathology: lessons from interferon-alpha. Biol Psychiatry 2004;56: 819-824.

22. Maninger N, Wolkowitz OM, Reus VI, Epel ES, Mellon SH. Neurobiological and neuropsychiatric effects of dehydroepiandrosterone (DHEA) and DHEA sulfate (DHEAS). Front Neuroendocrinol 2009;30:65-91.

23. Bottiglieri T. Homocysteine and folate metabolism in depression. Prog Neuropsychopharmacol Biol Psychiatry 2005;29:1103-1112.

24. Koponen H, Jokelainen J, Keinänen-Kiukaanniemi S, Kumpusalo E, Vanhala M. Metabolic syndrome predisposes to depressive symptoms: a population-based 7-year followup study. J Clin Psychiatry 2008;69:178-182.

25. Berr C, Lafont S, Debuire B, Dartigues JF, Baulieu EE. Relationships of dehydroepiandrosterone sulfate in the elderly with functional, psychological, and mental status, and short-term mortality: a French community-based study. Proc Natl Acad Sci U S A 1996;93:13410-13415.

26. Hahn HM, Yum TH, Shin YW, Kim KH, Yoon DJ, Chung KJ. A standardization study of Beck Depression Inventory in Korea. J Korean Neuropsychiatr Assoc 1986;25:487-500.

27. Bouman TK, Kok AR. Homogeneity of Beck's Depression Inventory (BDI): applying Rasch analysis in conceptual exploration. Acta Psychiatr Scand 1987;76:568-573.

28. Zheng P. Neuroactive steroid regulation of neurotransmitter release in the CNS: action, mechanism and possible significance. Prog Neurobiol 2009;89:134-152.

29. Dubrovsky BO. Steroids, neuroactive steroids and neuro- steroids in psychopathology. Prog Neuropsychopharmacol Biol Psychiatry 2005;29:169-192.

30. Traish AM, Kang HP, Saad F, Guay AT. Dehydroepiandrosterone (DHEA)--a precursor steroid or an active hormone in human physiology. J Sex Med 2011;8:29602982; quiz 2983.

31. Genud R, Merenlender A, Gispan-Herman I, Maayan R, Weizman A, Yadid G. DHEA lessens depressive-like behavior via GABA-ergic modulation of the mesolimbic system. Neuropsychopharmacology 2009;34:577-584.

32. Hu Q, Zhang SY, Liu F, Zhang YL, Zhu DM, Zang YY. Clinical significance of decreased protein expression of dehydroepiandrosterone sulfate in the development of depression: a meta-analysis. J Affect Disord 2015;174: 416-423.

33. Barrett-Connor E, von Mühlen D, Laughlin GA, Kripke A. Endogenous levels of dehydroepiandrosterone sulfate, but not other sex hormones, are associated with depressed mood in older women: the Rancho Bernardo study. J Am Geriatr Soc 1999;47:685-691.

34. Kurita H, Maeshima H, Kida S, Matsuzaka H, Shimano T, Nakano Y, et al. Serum dehydroepiandrosterone (DHEA) and DHEA-sulfate (S) levels in medicated patients with major depressive disorder compared with controls. J Affect Disord 2013;146:205-212.

35. Heuser I, Deuschle M, Luppa P, Schweiger U, Standhardt $\mathrm{H}$, Weber B. Increased diurnal plasma concentrations of dehydroepiandrosterone in depressed patients. J Clin Endocrinol Metab 1998;83:3130-3133.

36. Erdinçler D, Bugay G, Ertan T, Eker E. Depression and sex hormones in elderly women. Arch Gerontol Geriatr 2004;39:239-244.

37. Lennartsson AK, Kushnir MM, Bergquist J, Jonsdottir IH. DHEA and DHEA-S response to acute psychosocial stress in healthy men and women. Biol Psychol 2012;90:143-149.

38. Izawa S, Sugaya N, Shirotsuki K, Yamada KC, Ogawa N, Ouchi Y, et al. Salivary dehydroepiandrosterone secretion in response to acute psychosocial stress and its correlations with biological and psychological changes. Biol Psychol 2008; 79:294-298.

39. Rabkin JG, McElhiney MC, Rabkin R, McGrath PJ, Ferrando SJ. Placebo-controlled trial of dehydroepiandrosterone (DHEA) for treatment of nonmajor depression in patients with HIV/AIDS. Am J Psychiatry 2006;163:59-66.

40. Wolkowitz OM, Reus VI, Roberts E, Manfredi F, Chan T, Raum WJ, et al. Dehydroepiandrosterone (DHEA) treatment of depression. Biol Psychiatry 1997;41:311-318.

41. Bloch M, Schmidt PJ, Danaceau MA, Adams LF, Rubinow DR. Dehydroepiandrosterone treatment of midlife dysthymia. Biol Psychiatry 1999;45:1533-1541.

42. Dayal M, Sammel MD, Zhao J, Hummel AC, Vandenbourne $\mathrm{K}$, Barnhart KT. Supplementation with DHEA: effect on muscle size, strength, quality of life, and lipids. J Womens Health (Larchmt) 2005; 14:391-400.

43. Labrie F, Luu-The V, Labrie C, Simard J. DHEA and its transformation into androgens and estrogens in peripheral target tissues: intracrinology. Front Neuroendocrinol 2001; 22:185-212. 